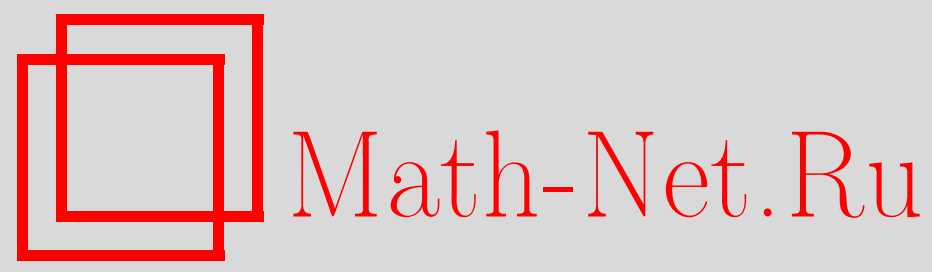

А. М. Гайсин, И. Д. Латыпов, Асимптотическое поведение суммы ряда Дирихле заданного роста на кривых, Матем. заметки, 2005, том 78, выпуск 1, 37-51

DOI: https://doi.org/10.4213/mzm2560

Использование Общероссийского математического портала Math-Net.Ru подразумевает, что вы прочитали и согласны с пользовательским соглашением http://www . mathnet.ru/rus/agreement

Параметры загрузки:

IP : 52.87 .193 .239

26 апреля 2023 г., $17: 17: 43$ 


\section{АСИМПТОТИЧЕСКОЕ ПОВЕДЕНИЕ СУММЫ РЯДА ДИРИХЛЕ ЗАДАННОГО РОСТА НА КРИВЫХ}

\section{А. М. Гайсин, И. Д. Латыпов}

Изучается связь между ростом и убыванием суммы целого ряда Дирихле конечного порядка по Ритту на произвольных кривых, уходящих в бесконечность. Для класса показателей, имеющих в некотором смысле регулярное распределение, найден критерий того, чтобы логарифм максимального члена был эквивалентен логарифму модуля суммы ряда Дирихле хотя бы на одной неограниченной последовательности точек кривой.

Библиографой: 11 названий.

Пусть $\Lambda=\left\{\lambda_{n}\right\}\left(0<\lambda_{n} \uparrow \infty\right)$ - последовательность, имеющая конечную верхнюю плотность

$$
D=\varlimsup_{n \rightarrow \infty} \frac{n}{\lambda_{n}}<\infty
$$

Через $D(\Lambda, R)$ обозначим класс всех функций $F$, представимых во всей плоскости рядами Дирихле

$$
F(s)=\sum_{n=1}^{\infty} a_{n} e^{\lambda_{n} s}, \quad s=\sigma+i t,
$$

и имеющих конечные порядки $\rho_{R}(F)$ по Ритту ( $R$-порядки). Напомним, что [1]

$$
\rho_{R}(F)=\varlimsup_{\sigma \rightarrow+\infty} \frac{\ln \ln M(\sigma)}{\sigma},
$$

где $M(\sigma)=\sup _{|t|<\infty}|F(\sigma+i t)|$.

Из условия (1) следует, что

$$
\lim _{n \rightarrow \infty} \frac{\ln n}{\lambda_{n}}=0
$$

так что ряд (2) сходится во всей плоскости абсолютно, а его сумма $F$ - целая функция [1]. Хорошо известно, что при условии (3) величина $\rho_{R}$ может быть вычислена по формуле [1]

$$
-\frac{1}{\rho_{R}}=\varlimsup_{n \rightarrow \infty} \frac{\ln \left|a_{n}\right|}{\lambda_{n} \ln \lambda_{n}} .
$$

Работа выполнена при финансовой поддержке Российского фонда фундаментальных исследований, грант № 99-01-00655. 
Рассмотрим произведение Вейерштрасса

$$
Q(z)=\prod_{n=1}^{\infty}\left(1-\frac{z^{2}}{\lambda_{n}^{2}}\right)
$$

Известно, что $Q$ - целая функция экспоненциального типа не выше $\pi D^{*}$, где $D^{*}$ - усредненная верхняя плотность последовательности $\Lambda$ :

$$
D^{*}=\varlimsup_{t \rightarrow \infty} \frac{N(t)}{t}, \quad N(t)=\int_{0}^{t} \frac{n(x)}{x} d x, \quad n(t)=\sum_{\lambda_{j \leqslant t}} 1 .
$$

Всегда $D^{*} \leqslant D \leqslant e D^{*}$ (см. [2]). Напомним также, что величина

$$
\delta=\varlimsup_{n \rightarrow \infty} \frac{1}{\lambda_{n}} \ln \left|\frac{1}{Q^{\prime}\left(\lambda_{n}\right)}\right|
$$

назьвается индексом конденсаиии последовательности $\Lambda$. Если $\inf _{n}\left(\lambda_{n+1}-\lambda_{n}\right)=$ $\tau>0$, то $\delta \leqslant 3[3-\ln (\tau D)] D<\infty[2$, гл. $2, \S 5]$. Наряду с рядом (2) введем в рассмотрение измененный ряд

$$
F^{*}(s)=\sum_{n=1}^{\infty} a_{n} Q^{\prime}\left(\lambda_{n}\right) e^{\lambda_{n} s}
$$

Так как $Q$ - целая функция экспоненциального типа, то ряд (6) также абсолютно сходится во всей плоскости, а $F^{*}$ - целая функция. При помощи формулы (4) проверяется, что если $F \in D(\Lambda, R)$, то $F^{*} \in D(\Lambda, R)$, причем $\rho_{R}^{*} \leqslant \rho_{R}$, где $\rho_{R}^{*}$ и $\rho_{R}-R$-порядки функций (6) и (2) соответственно. Если индекс конденсации $\delta<\infty$, то $\rho_{R}^{*}=\rho_{R}$.

Пусть $E \subset[0, \infty)$ - измеримое по Лебегу множество. Верхней $D E$ и нижней $d E$ плотностями множества $E$ назьваются величины

$$
D E=\varlimsup_{\sigma \rightarrow \infty} \frac{\operatorname{mes}(E \cap[0, \sigma])}{\sigma}, \quad d E=\varliminf_{\sigma \rightarrow \infty} \frac{\operatorname{mes}(E \cap[0, \sigma])}{\sigma} .
$$

В дальнейшем считаем, что все исключительные множества $E \subset[0, \infty)$, вне которых будут получены асимптотические оценки, представляют собой объединения отрезков вида $\left[a_{n}, a_{n}^{\prime}\right]$, где

$$
0<a_{1}<a_{1}^{\prime} \leqslant a_{2}<a_{2}^{\prime} \leqslant \cdots \leqslant a_{n}<a_{n}^{\prime} \leqslant \cdots .
$$

Через $L$ обозначим класс всех непрерьвных и неограниченно возрастающих на $\mathbb{R}_{+}=$ $[0, \infty)$ положительных функций $w=w(x)$. Пусть

$$
\begin{gathered}
W=\left\{w \in L: \int_{1}^{\infty} \frac{w(x)}{x^{2}} d x<\infty\right\}, \\
\underline{W}=\left\{w \in L: \sqrt{x} \leqslant w(x)=o(x \ln x), x \rightarrow \infty, \underline{\lim } \frac{1}{\ln x} \int_{1}^{x} \frac{w(t)}{t^{2}} d t=0\right\},
\end{gathered}
$$

a

$$
\bar{W}=\left\{w \in L: \sqrt{x} \leqslant w(x), \lim _{x \rightarrow \infty} \frac{1}{\ln x} \int_{1}^{x} \frac{w(t)}{t^{2}} d t=0\right\}
$$


Будем говорить, что последовательность $\left\{b_{n}\right\}, b_{n} \neq 0$ при $n \geqslant N, \bar{W}$-нормальна, если найдется функция $\theta \in L$ такая, что

$$
\lim _{x \rightarrow \infty} \frac{1}{\ln x} \int_{1}^{x} \frac{\theta(t)}{t^{2}} d t=0, \quad-\ln \left|b_{n}\right| \leqslant \theta\left(\lambda_{n}\right), \quad n \geqslant N .
$$

Пусть $\Gamma=\{\gamma\}-$ семейство кривых, где $\gamma-$ любая кривая, уходящая в бесконечность так, что если $s \in \gamma$ и $s \rightarrow \infty$, то $\operatorname{Re} s \rightarrow+\infty$.

Через $D(\Lambda)$ обозначим класс всех функций $F$, представимых во всей плоскости рядами Дирихле (2). Для $F \in D(\Lambda), \gamma \in \Gamma$ положим

$$
d(F ; \gamma) \stackrel{\text { def }}{=} \varlimsup_{s \in \gamma} \frac{\ln |F(s)|}{\ln M(\operatorname{Re} s)}
$$

где $M(\sigma)=\sup _{|t|<\infty}|F(\sigma+i t)|$.

Пусть, далее, $\mu(\sigma)$ и $\mu^{*}(\sigma)$ - максимальные члены рядов $(2)$ и (6) соответственно, т.е.

$$
\mu(\sigma)=\max _{n \geqslant 1}\left\{\left|a_{n}\right| e^{\lambda_{n} \sigma}\right\}, \quad \mu^{*}(\sigma)=\max _{n \geqslant 1}\left\{\left|a_{n}\right|\left|Q^{\prime}\left(\lambda_{n}\right)\right| e^{\lambda_{n} \sigma}\right\}
$$

В работе [3] доказана следующая

ТеоремА А. Пусть выполняется условие

$$
\sum_{n=1}^{\infty} \frac{1}{\lambda_{n}}<\infty
$$

Тогда для любой функиии $F \in D(\Lambda)$ справедлива оченка

$$
q(F) \leqslant d(F)
$$

Здесь

$$
d(F)=\inf _{\gamma \in \Gamma} d(F ; \gamma), \quad q(F)=\inf _{e} \varlimsup_{\substack{\sigma \in e \\ \sigma \rightarrow \infty}} \frac{\ln \mu^{*}(\sigma)}{\ln \mu(\sigma)}
$$

$d(F ; \gamma)$ - величина, определенная формулой (7), а точная нижняя грань и верхний предел в определении $q(F)$ вычисляются по всем множествам е $\subset \mathbb{R}_{+}$, кахдое из которых получается удалением из $\mathbb{R}_{+}$некоторой системы отрезков конечной суммарной длинны.

Пусть вьполняется условие (8). Тогда справедливы утверждения.

СлЕДСТВИЕ 1. Если при $\sigma \rightarrow \infty$ вне некоторого множсества $E \subset \mathbb{R}_{+}$конечной мерьи

$$
\ln \mu^{*}(\sigma)=(1+o(1)) \ln \mu(\sigma)
$$

$\operatorname{mo} q(F)=d(F)=1$. 
СлЕДСТВИЕ 2. Для любой челой функиии $F \in D(\Lambda)$ справедливь оценки

$$
0 \leqslant d(F) \leqslant 1
$$

Оценки (9) неулучшаемы [3]. Они указывают на связь между ростом и убыванием целой функции $F$ на каждой кривой $\gamma \in \Gamma$. Действительно, из оценки $0 \leqslant d(F ; \gamma)$ следует, что существуют функция $\varepsilon(r), \varepsilon \downarrow 0$ при $r \rightarrow \infty$, и последовательность $\left\{\xi_{n}\right\}, \xi_{n} \in \gamma$, такие, что при $\xi_{n} \rightarrow \infty$

$$
\ln \left|F\left(\xi_{n}\right)\right|>-\varepsilon\left(\left|\xi_{n}\right|\right) \ln M\left(\operatorname{Re} \xi_{n}\right) .
$$

Отметим, что аналогичная оценка для произвольных целых функций на фиксированном луче была установлена Бёрлингом в работе [4]: для любых $\varepsilon>0, \theta \in[0,2 \pi)(\theta$ фиксировано) множество

$$
\left\{r \in \mathbb{R}_{+}: \ln \left|f\left(r e^{i \theta}\right)\right|>-(1+\varepsilon) \ln M(r ; f)\right\}
$$

неограниченное, где $M(r ; f)=\max _{|z|=r}|f(z)|$.

Видим, что оценка (10) лучше соответствующей оценки Бёрлинга. Это объясняется тем, что в теореме А функция $F$ имеет специальный вид, а именно является суммой ряда Дирихле, для последовательности показателей которой вьполняется условие (8). Смысл оценки (10) в том, что при вьполнении условия (8) сумма целого ряда Дирихле (2) не может сколь угодно быстро убьвать на любой последовательности точек $\left\{\xi_{n}\right\}$, стремящейся к бесконечности вдоль кривой $\gamma$.

Цель статьи - доказать аналог теоремы А для функций $F$ из класса $D(\Lambda, R)$. Будет показано, что в этом случае условие (8) можно заменить на требование (теорема 1 )

$$
\varliminf_{x \rightarrow \infty} \frac{1}{\ln x} \sum_{\lambda_{n} \leqslant x} \frac{1}{\lambda_{n}}=0 .
$$

Для $\bar{W}$ - нормальной последовательности $\left\{Q^{\prime}\left(\lambda_{n}\right)\right\}$ - условие (11) необходимо и достаточно для того, чтобы для любой функции $F \in D(\Lambda, R)$ выполнялось равенство $d(F)=1$ ( теорема 2$)$.

1. Вспомогательные факты. В статье будет по существу использован один из вариантов следующей теоремы из работы [5].

ТЕорема (Борель-Неванлинна). Пусть на интервале $\left[r_{0}, \infty\right)$ задана неубъвающая непрерывная функиия $u(r), u(r) \rightarrow \infty$ nрu $r \rightarrow \infty$.

Пусть $\varphi(u)$ - положительная, невозрастающая и непрерывная на интервале $\left[u_{0}, \infty\right)\left(u_{0}=u\left(r_{0}\right)\right)$ функиия, $\varphi(u) \rightarrow 0$ при $u \rightarrow \infty$, причем

$$
\int_{u_{0}}^{\infty} \varphi(u) d u<\infty .
$$

Тогда для всех $r \geqslant r_{0}$, кроме, возмохсно, мнохества конечной меры, выполняется оченка

$$
u(r+\varphi(u(r)))<u(r)+1 .
$$

Имеет место следующая лемма типа Бореля-Неванлинны. 
ЛЕмма 1. Пусть $и(\sigma)$ - неубивающая непрерывная на $[0, \infty)$ функиия, $u(\sigma) \rightarrow \infty$ при $\sigma \rightarrow \infty$. Пусть $w \in L$,

$$
\frac{w(x)}{x \ln x}=o(1), \quad x \rightarrow \infty .
$$

Если $v=v(\sigma)$ - решение уравнения

$$
w(v)=e^{u(\sigma)}
$$

а для некоторой последовательности $\left\{\tau_{j}\right\}\left(0<\tau_{j} \uparrow \infty\right)$ при $\tau_{j} \rightarrow \infty$

$$
\ln v\left(\tau_{j}\right)=O\left(\tau_{j}\right)
$$

$u$

$$
\lim _{\tau_{j} \rightarrow \infty} \frac{1}{\tau_{j}} \int_{1}^{v\left(\tau_{j}\right)} \frac{w(t)}{t^{2}} d t=0,
$$

то при $\sigma \rightarrow \infty$ вне некоторого мнохсества $E \subset[0, \infty)$,

$$
\operatorname{mes}\left(E \cap\left[0, \tau_{j}\right]\right)=o\left(\tau_{j}\right)
$$

имеет место асимптотическая оченка

$$
u\left(\sigma+\frac{w(v(\sigma))}{v(\sigma)}\right)<u(\sigma)+o(1)
$$

Лемма 1 доказана в работе [6].

ЗАмечАниЕ. Найдется функция $w^{*}(t)=\beta(t) w(t)(0<\beta(t) \uparrow \infty, t \rightarrow \infty)$ из класса $L$, удовлетворяющая условиям (12), (15) леммы 1. С учетом (14) показано, что [6]

$$
\operatorname{mes}\left(E \cap\left[0, \tau_{j}\right]\right) \leqslant o\left(\ln v\left(\tau_{j}\right)\right)+4 \int_{v\left(\tau_{1}\right)}^{v\left(\tau_{j}\right)} \frac{w^{*}(t)}{t^{2}} d t=o\left(\tau_{j}\right) .
$$

Оценка (17) будет использована при доказательстве теоремы 1.

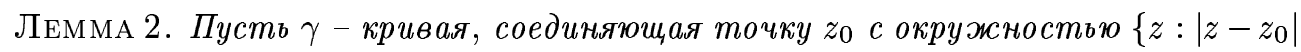
$=R\}$, состоящая из конечного числа кусочно-гладких жсордановых кривых, а $g(z)-$ функиия, аналитическая в круге $D\left(z_{0} ; R\right)=\left\{z:\left|z-z_{0}\right|<R\right\}$ и непрерывная в ее замыкании $\bar{D}\left(z_{0} ; R\right)$. Пусть

$$
M=\max _{\bar{D}\left(z_{0} ; R\right)}|g(t)|, \quad m=\max _{\gamma}|g(t)|
$$

Тогда при $0<\beta \leqslant 1 / 5$ для всех $z$ из круга $\bar{D}\left(z_{0} ; \beta R\right)$ верна оченка

$$
|g(z)| \leqslant m^{1-2 \beta} M^{2 \beta} .
$$

Лемма 2 доказана в работе [3].

2. Основные результаты. Сформулируем основные результаты статьи. 
ТЕОРема 1. Пусть последовательность $\Lambda$ имеет конечную верхнюю плотность. Если выполняется условие (11), то для любой функиии $F \in D(\Lambda, R)$ и любого $\beta$, $0<\beta \leqslant 1 / 5$, существует мнохсество $E_{\beta}, d E_{\beta}=0$, такое, что справедлива оченка

$$
\varlimsup_{\substack{\sigma \in e_{\beta} \\ \sigma \rightarrow \infty}} \frac{\ln \mu^{*}(\sigma)}{\ln \mu(\sigma)} \leqslant 2 \beta+(1-2 \beta) d(F ; \gamma),
$$

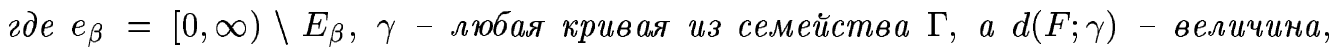
определенная формулой (7).

СЛЕДСТВИЕ. Пусть последовательность $\Lambda$ имеет конечную верхнюю плотность. Если выполняется условие (11), то для любой иелой функции $F \in D(\Lambda, R)$ справедливы оценки

$$
0 \leqslant d(F) \leqslant 1
$$

əде $d(F)=\inf _{\gamma \in \Gamma} d(F, \gamma)$.

ЗАмЕчАниЕ. Если в теореме 1 вместо условия (11) вьполняется более сильное требование

$$
\lim _{x \rightarrow \infty} \frac{1}{\ln x} \sum_{\lambda_{n} \leqslant x} \frac{1}{\lambda_{n}}=0,
$$

TO

$$
q(F) \leqslant d(F)
$$

где

$$
d(F)=\inf _{\gamma \in \Gamma} d(F, \gamma), \quad q(F)=\inf _{e} \varlimsup_{\substack{\sigma \in e \\ \sigma \rightarrow \infty}} \frac{\ln \mu^{*}(\sigma)}{\ln \mu(\sigma)}
$$

а точная нижняя грань и верхний предел в определении $q(F)$ вычисляются по всем множествам $e \subset \mathbb{R}_{+}$, каждое из которых получается удалением из $\mathbb{R}_{+}$некоторой системы отрезков, объединение которых имеет нулевую плотность. Дело в том, что в этом случае все асимптотические оценки, в том числе и (19), полученные в ходе доказательства теоремы 1 , будут справедливы вне некоторых множеств нулевой плотности. Поэтому (21) есть следствие оценки (19).

Если при $\sigma \rightarrow \infty$ вне некоторого множества $E$ нулевой плотности

$$
\ln \mu^{*}(\sigma)=(1+o(1)) \ln \mu(\sigma)
$$

то из $(21)$ видно, что $q(F)=d(F)=1$. Действительно, объединение двух множеств нулевой плотности имеет также нулевую плотность, $0 \leqslant q(F) \leqslant d(F) \leqslant 1$. Поскольку $(22)$ справедливо вне множества нулевой плотности, то, очевидно, $q(F)=1$. Следовательно, $d(F)=1$.

Заметим, что если (22) имеет место вне некоторого множества нулевой нижней плотности, равенство $d(F)=1$ получается из оценки (19) теоремы 1 . В условиях теоремы 1 аналогичное определение $q(F)$ некорректно, поскольку объединение двух множеств нулевой нижней плотности не обязано иметь нулевую нижнюю плотность. Поэтому в теореме ограничиваемся лишш оценкой (19). 
ТЕОРема 2. Пусть последовательность $\Lambda$ имеет конечную верхнюю плотность. Предположим, что последовательность $\left\{Q^{\prime}\left(\lambda_{n}\right)\right\} \bar{W}$-нормальна. Для того чтобы для любой функиии $F \in D(\Lambda, R)$ выполнялось равенство $d(F)=1$, необходимо и достаточно, чтобы выполнялось условие (11).

Отметим следуюшее. Пусть последовательность $\Lambda$ удовлетворяет условию (20). Если дажепоследовательность $\left\{Q^{\prime}\left(\lambda_{n}\right)\right\}$ не является $\bar{W}$-нормальной, всегда имеется функция $F \in D(\Lambda, R)$, для которой $q(F)=d(F)=1$ [3]. В этом главный смысл оценки (21).

СлеДСтвиЕ. Пусть $P=\left\{p_{n}\right\}-$ возрастающая последовательность натуральных чисел,

$$
Q(z)=\prod_{n=1}^{\infty}\left(1-\frac{z^{2}}{p_{n}^{2}}\right)
$$

причем последовательность $\left\{Q^{\prime}\left(p_{n}\right)\right\} \bar{W}$-нормальна.

Для того, чтобы для любой челой функции $f$ конечного порядка, имеющей вид

$$
f(z)=\sum_{n=1}^{\infty} a_{n} z^{p_{n}}, \quad a_{n} \neq 0,
$$

выполнялось равенство $d(f)=1$, необходимо и достаточно, чтобы выполнялось условие

$$
\varliminf_{x \rightarrow \infty} \frac{1}{\ln x} \sum_{p_{n} \leqslant x} \frac{1}{p_{n}}=0 .
$$

Здесь $d(f)=\inf _{\gamma} d(f, \gamma), \gamma-$ любая кривая, уходящая в бесконечность,

$$
d(f, \gamma)=\varlimsup_{\substack{z \in \gamma \\ z \rightarrow \infty}} \frac{\ln |f(z)|}{\ln M(|z| ; f)} .
$$

Следствие легко вытекает из теоремы 2 , если сделать замену $z=e^{s}$. Действительно,

$$
F(s)=f\left(e^{s}\right)=\sum_{n=1}^{\infty} a_{n} e^{p_{n} s}
$$

- целая функция конечного $R$-порядка. Следовательно, $d(f)=d(F)$, и все следует из теоремы 2.

Пусть целая функция $f$ конечного порядка имеет вид (23). Если последовательность $P=\left\{p_{n}\right\}$ имеет нулевую плотность, то $d(f)=1$. Этот факт впервые был установлен $\Gamma$. Пойа в работе [7]. Заметим, что равенство $d(f)=1$ легко вытекает также из теоремы 2 (см. следствие). Действительно, так как плотность последовательности $P=\left\{p_{n}\right\}$ равна нулю, то

$$
\lim _{x \rightarrow \infty} \frac{1}{\ln x} \sum_{p_{n} \leqslant x} \frac{1}{p_{n}}=0 .
$$

Следовательно, условие (24) подавно вьполнено. Более того, индекс конденсации $\delta=0$ [2]. Это означает, что существует функция $\theta \in L, \theta(x)=o(x)$, такая, что

$$
-\ln \left|Q^{\prime}\left(p_{n}\right)\right| \leqslant \theta\left(p_{n}\right), \quad n \geqslant 1 .
$$


Ясно, что $\theta \in \bar{W}$. Значит, последовательность $\left\{Q^{\prime}\left(p_{n}\right)\right\} \bar{W}$-нормальна. Но тогда, как следует из теоремы $2, d(f)=1$.

В случае, когда $\gamma-$ луч $\mathbb{R}_{+}, \Lambda=\left\{\lambda_{n}\right\}$ - последовательность, имеющая нулевую плотность, причем $\lambda_{n+1}-\lambda_{n} \geqslant h>0, n \geqslant 1$, результат Пойа из [7] перенесен М. Н. Шереметой на ряды Дирихле $(2)$ из класса $D(\Lambda, R)[8]$.

В работе [9] показано, что если $\delta=0$ и $\Delta=0(\delta$-индекс конденсации, $\Delta-$ плотность последовательности $\Lambda$ ), то для любой функции $F \in D(\Lambda, R)$ и любой кривой $\gamma$ из Г выполняется равенство $d(F ; \gamma)=1$. Если кривая содержится в некоторой горизонтальной полосе конечной ширины, аналогичная оценка установлена в статье [10].

Теорема 2 является обобщением всех перечисленных вьше результатов, и в классе целых функций $F \in D(\Lambda, R)$ она носит законченный характер.

3. Доказательство теоремы 1. Имеем

$$
\sum_{\lambda_{n} \leqslant x} \frac{1}{\lambda_{n}}=\int_{0}^{x} \frac{d n(t)}{t}=\frac{n(x)}{x}+\int_{0}^{x} \frac{n(t)}{t^{2}} d t=\frac{N(x)}{x}+\frac{n(x)}{x}+\int_{0}^{x} \frac{N(t)}{t^{2}} d t .
$$

Следовательно,

$$
\left|\sum_{\lambda_{n} \leqslant x} \frac{1}{\lambda_{n}}-\int_{0}^{x} \frac{N(t)}{t^{2}} d t\right| \leqslant \frac{n(x)}{x}+\frac{N(x)}{x} .
$$

Но функции $n(x), N(x)$ имеют одинаковые порядки и типы [11]. В данном случае

$$
\varlimsup_{x \rightarrow \infty} \frac{n(x)}{x}<\infty, \quad \varlimsup_{x \rightarrow \infty} \frac{N(x)}{x}<\infty .
$$

Следовательно,

$$
\left|\sum_{\lambda_{n} \leqslant x} \frac{1}{\lambda_{n}}-\int_{0}^{x} \frac{N(t)}{t^{2}}\right| \leqslant a<\infty .
$$

Значит, из (11) получаем, что

$$
\varliminf_{x \rightarrow \infty} \frac{1}{\ln x} \int_{0}^{x} \frac{N(t)}{t^{2}} d t=0 .
$$

Положим $w(t)=\max (\sqrt{t}, N($ et $))$. Ясно, что $w \in \underline{W}$. Тогда существует функция $w^{*}(x) \in \underline{W}$ такая, что $w^{*}(x)=\beta(x) w(x)(0<\beta(x) \uparrow \infty)$.

Пусть $v=v(\sigma)$ - решение уравнения

$$
w_{1}(v)=3 \ln \mu(\sigma)
$$

где $w_{1}(x)=\sqrt{\beta(x)} w(x)$. Положим

$$
R_{v}=\sum_{\lambda_{j}>v}\left|a_{j}\right| e^{\lambda_{j} \sigma}, \quad h=\frac{w_{1}(v)}{v}, \quad v=v(\sigma) .
$$

Так как последовательность $\Lambda$ имеет конечную верхнюю плотность, то

$$
\sum_{n=1}^{\infty} \frac{1}{\lambda_{n}^{2}}<\infty
$$


Следовательно,

$$
R_{v} \leqslant \mu(\sigma+h) \sum_{\lambda_{n}>v} e^{-\lambda_{n} h} \leqslant \mu(\sigma+h) C \exp \left(\max _{t \geqslant v} \varphi(t)\right),
$$

где

$$
\varphi(t)=2 \ln t-h t, \quad C=\sum_{n=1}^{\infty} \frac{1}{\lambda_{n}^{2}} .
$$

Поскольку $\varphi^{\prime}(t)=0$ в точке $t_{0}$,

$$
t_{0}=\frac{2}{h}=2 \frac{v}{w_{1}(v)} \leqslant 2 \frac{v}{\sqrt{v}}=2 \sqrt{v}<v=v(\sigma)
$$

при $\sigma \geqslant \sigma_{0}$, то при $\sigma \rightarrow \infty$

$$
R_{v} \leqslant C \mu(\sigma+h) \exp [-v(1+o(1)) h]=C \mu(\sigma+h) \exp \left[-(1+o(1)) w_{1}(v)\right] .
$$

Положим $u(\sigma)=\ln 3+\ln \ln \mu(\sigma)$. Поскольку $\mu(\sigma) \leqslant M(\sigma)$, а $F \in D(\Lambda, R)$, то

$$
\varlimsup_{\sigma \rightarrow \infty} \frac{u(\sigma)}{\sigma}<\infty
$$

Следовательно, с учетом (25) при $\sigma \geqslant \sigma_{0}$ имеем

$$
\ln w_{1}(v(\sigma))=u(\sigma) \leqslant A \sigma, \quad 0<A<\infty .
$$

Но $\sqrt{x} \leqslant w_{1}(x)$. Следовательно,

$$
\frac{1}{\sigma} \leqslant \frac{2 A}{\ln v(\sigma)}, \quad \sigma \geqslant \sigma_{0}
$$

Далее, поскольку $w^{*} \in \underline{W}$, то

$$
\lim _{x \rightarrow \infty} \frac{w^{*}(x)}{x \ln x}=0
$$

а для некоторой последовательности $\left\{t_{j}\right\}\left(0<t_{j} \uparrow \infty\right)$

$$
\lim _{t_{j} \rightarrow \infty} \frac{1}{\ln t_{j}} \int_{1}^{t_{j}} \frac{w^{*}(t)}{t^{2}} d t=0 .
$$

Более того, если $\tau_{j}$ - корень уравнения $v(\sigma)=t_{j}$, то из $(27),(29)$ получаем, что

$$
\lim _{\tau_{j} \rightarrow \infty} \frac{1}{\tau_{j}} \int_{1}^{v\left(\tau_{j}\right)} \frac{w^{*}(t)}{t^{2}} d t=0
$$

Далее, из (27), в частности, имеем $\ln v\left(\tau_{j}\right)=O\left(\tau_{j}\right)$ при $\tau_{j} \rightarrow \infty$. Так как $w_{1}(x) \leqslant w^{*}(x)$, видим, что для пары функций $u$ и $w_{1}$ все условия леммы 1 вьполнены. Поэтому, применяя эту лемму и учитывая при этом $(17),(27),(28)$, а также то, что $\ln v\left(\tau_{j}\right)=O\left(\tau_{j}\right)$ при $\tau_{j} \rightarrow \infty$, вне некоторого множества $E_{\beta}^{\prime} \subset[0, \infty)$,

$$
\operatorname{mes}\left(E_{\beta}^{\prime} \cap\left[0, \tau_{j}\right]\right) \leqslant o\left(\ln v\left(\tau_{j}\right)\right)+4 \int_{v\left(\tau_{1}\right)}^{v\left(\tau_{j}\right)} \frac{w^{*}(t)}{t^{2}} d t=o\left(\tau_{j}\right), \quad \tau_{j} \rightarrow \infty,
$$


при $\sigma \rightarrow \infty$ получаем, что

$$
\mu\left(\sigma+\left(\beta^{-1}+1\right) h(\sigma)\right)=\mu(\sigma)^{1+o(1)}, \quad 0<\beta \leqslant 1 .
$$

Следовательно, из $(26),(31)$ получаем, что при $\sigma \rightarrow \infty$ вне $E_{\beta}^{\prime}$

$$
R_{v} \leqslant C \mu(\sigma)^{1+o(1)} \exp \left[-w_{1}(v)(1+o(1))\right]=\mu(\sigma)^{-2(1+o(1))}
$$

Пусть

$$
P_{a}(s)=\sum_{\lambda_{n} \leqslant a} a_{n} e^{\lambda_{n} s}, \quad s=\sigma+i t
$$

Тогда [2]

$$
a_{n}=e^{-\alpha \lambda_{n}} \frac{1}{2 \pi i} \int_{C} \varphi_{n}(t) P_{a}(t+\alpha) d t
$$

где

$$
\varphi_{n}(t)=\frac{1}{q_{a}^{\prime}\left(\lambda_{n}\right)} \int_{0}^{\infty} \frac{q_{a}(\lambda)}{\lambda-\lambda_{n}} e^{-\lambda t} d \lambda, \quad q_{a}(\lambda)=\prod_{\lambda_{n} \leqslant a}\left(1-\frac{\lambda^{2}}{\lambda_{n}^{2}}\right)
$$

а $C$ - любой замкнутьй контур, охватьвающий сопряженную диаграмму $q_{a}$, т.е. начало координат.

Положим $a=v(\sigma)$, а в качестве $C$ возьмем контур $\{t:|t|=h(\sigma)\}$. Пусть $\alpha=\sigma+i \tau$, где $\tau$ такое, что $\alpha \in \gamma$. Поскольку для $\lambda_{n} \leqslant v(\sigma)$

$$
\frac{1}{\left|q_{v}^{\prime}\left(\lambda_{n}\right)\right|} \leqslant \frac{1}{\left|Q^{\prime}\left(\lambda_{n}\right)\right|}, \quad n \geqslant 1
$$

из $(33),(34)$ получаем, что для любого $\lambda_{n} \leqslant v(\sigma)$

$$
\begin{aligned}
\left|a_{n}\right|\left|Q^{\prime}\left(\lambda_{n}\right)\right| e^{\lambda_{n} \sigma} \leqslant & h(\sigma)\left[\max _{|\xi-\alpha| \leqslant h(\sigma)}|F(\xi)|+\sum_{\lambda_{j}>v}\left|a_{j}\right| e^{\lambda_{j}(\sigma+h(\sigma))}\right] \\
& \times \int_{0}^{\infty} M\left(r+2 ; q_{v}\right) e^{-h(\sigma) r} d r .
\end{aligned}
$$

Далее, учитьвая определения величин $v=v(\sigma), h=h(\sigma)$, при $\sigma \rightarrow \infty$ имеем

$$
\begin{aligned}
\ln M\left(R ; q_{v}\right) & =n(v) \ln \left(1+\frac{R^{2}}{v^{2}}\right)+2 R^{2} \int_{0}^{v} \frac{n(t)}{t\left(t^{2}+R^{2}\right)} d t \\
& \leqslant \frac{n(v)}{v} R+2 N(v)=o(1) h(\sigma) R+o(1) \ln \mu(\sigma) .
\end{aligned}
$$

Следовательно, учитывая (31) и оценку типа (32), из (35) получаем, что для всех $\lambda_{n} \leqslant$ $v(\sigma)$ при $\sigma \rightarrow \infty$ вне $E_{\beta}^{\prime}$

$$
\left|a_{n}\right|\left|Q^{\prime}\left(\lambda_{n}\right)\right| e^{\lambda_{n} \sigma} \leqslant \mu(\sigma)^{-2(1+o(1))}+\mu(\sigma)^{o(1)} \max _{|\xi-\alpha| \leqslant h(\sigma)}|F(\xi)| .
$$


Пусть $\mu^{*}(\sigma)$ - максимальный член ряда

$$
F^{*}(s)=\sum_{n=1}^{\infty} a_{n} Q^{\prime}\left(\lambda_{n}\right) e^{\lambda_{n} s}
$$

Tak как

$$
\varlimsup_{n \rightarrow \infty} \frac{\ln \left|Q^{\prime}\left(\lambda_{n}\right)\right|}{\lambda_{n}}<\infty
$$

данньй ряд абсолютно сходится во всей комплексной плоскости и его сумма имеет конечньй $R$-порядок.

Пусть $p=p(\sigma)$ - решение уравнения $w_{1}(p)=2 \ln \mu^{*}(\sigma)$, а

$$
R_{p}^{*}=\sum_{\lambda_{n}>p}\left|a_{n}\right|\left|Q^{\prime}\left(\lambda_{n}\right)\right| e^{\lambda_{n} \sigma}, \quad p=p(\sigma) .
$$

Поскольку $F^{*} \in D(\Lambda, R)$, то

$$
\varlimsup_{\sigma \rightarrow \infty} \frac{u^{*}(\sigma)}{\sigma}<\infty, \quad u^{*}(\sigma)=\ln 2+\ln \ln \mu^{*}(\sigma) .
$$

Поэтому, применяя лемму 1 , из тех же рассуждений, при помощи которых была получена оценка для $R_{v}$, получаем, что

$$
R_{p}^{*} \leqslant C \mu^{*}(\sigma)^{-2(1+o(1))}, \quad C=\sum_{n=1}^{\infty} \frac{1}{\lambda_{n}^{2}},
$$

если $\sigma \rightarrow \infty$ вне некоторого множества $E_{1} \subset[0, \infty)\left(E_{1}\right.$ от $\beta$ не зависит),

$$
\operatorname{mes}\left(E_{1} \cap\left[0, x_{j}\right]\right) \leqslant o\left(\ln p\left(x_{j}\right)\right)+4 \int_{p\left(x_{1}\right)}^{p\left(x_{j}\right)} \frac{w^{*}(t)}{t^{2}} d t, \quad x_{j} \rightarrow \infty .
$$

Здесь $x_{j}$ - решение уравнения $p(\sigma)=t_{j}$, a $\left\{t_{j}\right\}$ - последовательность, фигурирующая в условии (29). Отсюда следует, что $\lambda_{k(\sigma)} \leqslant p(\sigma)$, если $\sigma \geqslant \sigma_{1}, \sigma \notin E_{1}$. Здесь $k(\sigma)-$ центральньй индекс ряда (37). Следовательно, при $\sigma \rightarrow \infty$ вне $E_{1}$

$$
\mu^{*}(\sigma) \leqslant \mu(\sigma) e^{w(p(\sigma))}=\mu(\sigma)\left[\mu^{*}(\sigma)\right]^{o(1)},
$$

т.е.

$$
(1+o(1)) \ln \mu^{*}(\sigma) \leqslant \ln \mu(\sigma) .
$$

Рассмотрим последовательность $\left\{\tau_{j}^{*}\right\}$, где $\left\{\tau_{j}^{*}\right\}=\min \left(\tau_{j}, x_{j}\right)$. Поскольку $t_{j}=$ $p\left(x_{j}\right)=v\left(\tau_{j}\right)$, из оценок типа $(27)$ получаем, что

$$
\frac{1}{\tau_{j}^{*}} \leqslant \frac{B}{\ln t_{j}}, \quad j \geqslant 1 .
$$

Следовательно, если $E_{\beta}=E_{\beta}^{\prime} \cup E_{1}$, то, учитьвая $(30),(38)$, при $\tau_{j}^{*} \rightarrow \infty$ получаем, что

$$
\begin{aligned}
\frac{\operatorname{mes}\left(E_{\beta} \cap\left[0, \tau_{j}^{*}\right]\right)}{\tau_{j}^{*}} & \leqslant \frac{B}{\ln t_{j}}\left(\operatorname{mes}\left(E_{\beta}^{\prime} \cap\left[0, \tau_{j}\right]\right)+\operatorname{mes}\left(E_{1} \cap\left[0, x_{j}\right]\right)\right) \\
& \leqslant \frac{B}{\ln t_{j}}\left[o\left(\ln t_{j}\right)+8 \int_{t_{1}}^{t_{j}} \frac{w^{*}(t)}{t^{2}} d t\right]=o(1) .
\end{aligned}
$$

Поскольку $R_{v} \leqslant 1, R_{p}^{*} \leqslant 1(v=v(\sigma), p=p(\sigma))$ при $\sigma \geqslant \sigma_{2}, \sigma \notin E_{\beta}$, то, очевидно,

1) $\lambda_{\nu(\sigma)} \leqslant v(\sigma)$

2) $\lambda_{k(\sigma)} \leqslant p(\sigma)$ 
где $\nu(\sigma), k(\sigma)$ - центральные индексы рядов (2) и (37).

Далее, для $\lambda_{n} \leqslant p(\sigma)$

$$
\left|Q^{\prime}\left(\lambda_{n}\right)\right| \leqslant \frac{2}{\lambda_{n}} \prod_{\substack{j \neq n \\ \lambda_{j} \leqslant p(\sigma)}}\left|1-\frac{\lambda_{n}^{2}}{\lambda_{j}^{2}}\right| \leqslant \frac{2}{\lambda_{1}} \prod_{\lambda_{j} \leqslant p(\sigma)}\left|1+\frac{\lambda_{n}^{2}}{\lambda_{j}^{2}}\right| .
$$

Но при $\lambda_{n} \leqslant p(\sigma)$ и $\sigma \rightarrow \infty$

$$
\begin{aligned}
\ln \prod_{\lambda_{j} \leqslant p(\sigma)}\left(1+\frac{\lambda_{n}^{2}}{\lambda_{j}^{2}}\right) & =n(p) \ln \left(1+\frac{\lambda_{n}^{2}}{p^{2}}\right)+2 p^{2} \int_{0}^{p} \frac{n(t)}{t\left(t^{2}+p^{2}\right)} d t \\
& \leqslant n(p) \frac{\lambda_{n}}{p}+2 N(p) \leqslant 3 N(e p)=o\left(\ln \mu^{*}(\sigma)\right) .
\end{aligned}
$$

Так что для $\lambda_{n} \leqslant p(\sigma)$ и $\sigma \rightarrow \infty$

$$
\left|Q^{\prime}\left(\lambda_{n}\right)\right| \leqslant\left[\mu^{*}(\sigma)\right]^{(o(1))} .
$$

Следовательно,

$$
\mu^{*}(\sigma)=\left|a_{k} Q^{\prime}\left(\lambda_{k}\right)\right| e^{\lambda_{k} \sigma} \leqslant \mu(\sigma)\left[\mu^{*}(\sigma)\right]^{o(1)},
$$

где $p=p(\sigma), k=k(\sigma)$. Значит, $(1+o(1)) \ln \mu^{*}(\sigma) \leqslant \ln \mu(\sigma)$ при $\sigma \rightarrow \infty$. Так что $w_{1}(p)=2 \ln \mu^{*}(\sigma)<3 \ln \mu(\sigma)=w_{1}(v), \sigma \geqslant \sigma_{3}$. Это означает, что $\lambda_{k(\sigma)} \leqslant p(\sigma)<v(\sigma)$ при $\sigma \geqslant \sigma_{3}, \sigma \notin E_{\beta}$. С учетом этого из (36) получаем, что при $\sigma \rightarrow \infty$ вне $E_{\beta}=E_{\beta}^{\prime} \cup E_{1}$

$$
\mu^{*}(\sigma)<1+\mu(\sigma)^{o(1)} \max _{|\xi-\alpha| \leqslant h(\sigma)}|F(\xi)|
$$

где $\alpha=\sigma+i \tau, \alpha \in \gamma$.

Дальнейшие рассуждения основаны на лемме 2.

Пусть $\gamma(\alpha)$ - часть кривой, содержащейся в круге $\bar{D}\left(\alpha ; h \beta^{-1}\right), 0<\beta \leqslant 1 / 5, h=h(\sigma)$. Она не обязательно кусочно-гладкая и жорданова. Поэтому поступаем следующим образом .

Пусть $\alpha_{1}$ - какая-нибудь точка кривой $\gamma(\alpha)$, принадлежашая окружности $\partial \bar{D}(\alpha$; $\left.h \beta^{-1}\right)$. Соединим точку $\alpha_{1}$ с $\alpha$ ломаной $l(\alpha)$ без самопересечений и такой , что

$$
\rho(\xi)=\inf _{z \in \gamma(\alpha)}|F(z)-F(\xi)| \leqslant m_{\alpha}
$$

где $\xi \in l(\alpha), m_{\alpha}=\max _{\gamma(\alpha)}|F(t)|$. Это можно сделать. Действительно, для любого $z \in \gamma(\alpha)$ существует окрестность $D\left(z ; \nu_{z}\right), \nu_{z}>0$, такая, что

$$
|F(z)-F(\xi)| \leqslant m_{\alpha}
$$

для любого $\xi \in D\left(z ; \nu_{z}\right)$. Поскольку $\gamma(\alpha)$ - компакт, по лемме Гейне-Бореля из покрытия $\bigcup_{z \in \gamma(\alpha)} D\left(z ; \nu_{z}\right)$ можно выделить конечное подпокрытие: $D\left(z_{0} ; \nu_{0}\right), D\left(z_{1} ; \nu_{1}\right), \ldots$, $D\left(z_{n} ; \nu_{n}\right)$, где $z_{i} \in \gamma(\alpha), \nu_{i}>0, i=0,1, \ldots, n$.

Можем считать, что $z_{0}=\alpha, z_{n}=\alpha_{1}$. Соединим между собой центры всех попарнопересекающихся кружков $D\left(z_{i} ; \nu_{i}\right), i=0,1, \ldots, n$, отрезками прямых. Отбрасывая, если 
это необходимо, конечное число отрезков, получим жорданову ломаную $l(\alpha)$, соединяющую точки $\alpha$ и $\alpha_{1}$. Заметим, что $l(\alpha)$ целиком содержится в объединении кружков $D\left(z_{i} ; \nu_{i}\right), i=0,1, \ldots, n$. Осталось проверить оценку (41).

Пусть $\xi \in l(\alpha)$. Тогда существует круг $D\left(z_{i} ; \nu_{i}\right)$, содержащий эту точку. Поскольку $\left|F\left(z_{i}\right)-F(\xi)\right| \leqslant m_{\alpha}$, то $\rho(\xi) \leqslant m_{\alpha}$.

Вернемся теперь к оценке (40). Применяя лемму 2 для круга $\bar{D}\left(z_{0} ; \beta^{-1} h\right)$ и кривой $l(\alpha)$, получаем

$$
\begin{aligned}
\max _{|\xi-\alpha| \leqslant h(\sigma)}|F(\xi)| & \leqslant \max _{l(\alpha)}|F(t)|^{1-2 \beta} \max _{|t-\alpha| \leqslant \beta^{-1} h(\sigma)}|F(t)|^{2 \beta} \\
& \leqslant\left|F\left(\xi_{\alpha}\right)\right|^{1-2 \beta} M^{2 \beta}\left(\sigma+\beta^{-1} h(\sigma)\right), \quad \xi_{\alpha} \in l(\alpha) .
\end{aligned}
$$

Но из (41) следует, что

$$
\rho\left(\xi_{\alpha}\right)=\left|F\left(\xi_{\alpha}\right)-F\left(z_{\alpha}\right)\right| \leqslant m_{\alpha}
$$

для некоторой точки $z_{\alpha} \in \gamma(\alpha)$. Следовательно,

$$
\max _{|\xi-\alpha| \leqslant h(\sigma)}|F(\xi)| \leqslant\left[2 m_{\alpha}\right]^{1-2 \beta} M^{2 \beta}\left(\sigma+\beta^{-1} h(\sigma)\right)=\left(2\left|F\left(z_{\alpha}^{\prime}\right)\right|\right)^{1-2 \beta} M^{2 \beta}\left(\sigma+\beta^{-1} h(\sigma)\right),
$$

где $z_{\alpha}^{\prime} \in \gamma_{\alpha}$.

Применяя лемму 1, имеем (см. (31))

$$
\begin{aligned}
\mu(\sigma) & \leqslant M(\sigma) \leqslant M\left(\sigma+\beta^{-1} h(\sigma)\right) \leqslant \sum_{n=1}^{\infty}\left|a_{n}\right| e^{\lambda_{n}\left(\sigma+\beta^{-1} h(\sigma)\right)} \\
& \leqslant \mu\left(\sigma+\left(1+\beta^{-1}\right) h(\sigma)\right)\left[n(v(\sigma))+\sum_{\lambda_{n}>v(\sigma)} e^{-h(\sigma) \lambda_{n}}\right]<\mu(\sigma)^{1+o(1)}
\end{aligned}
$$

когда $\sigma \rightarrow \infty$ вне множества $E_{\beta}^{\prime}$. Следовательно, с учетом оценок $(42),(43)$ из (40) получаем, что при $\sigma \rightarrow \infty$ вне множества $E_{\beta}=E_{\beta}^{\prime} \cup E_{1}$ нулевой нижней плотности

$$
(1+o(1)) \mu^{*}(\sigma) \leqslant 2\left|F\left(z_{\alpha}^{\prime}\right)\right|^{1-2 \beta} \mu(\sigma)^{(1+o(1)) 2 \beta}, \quad z_{\alpha}^{\prime} \in \gamma(\alpha)
$$

Следовательно, при $\sigma \rightarrow \infty$ вне $E_{\beta}$

$$
\frac{\ln \mu^{*}(\sigma)}{\ln \mu(\sigma)} \leqslant(1+o(1)) 2 \beta+(1-2 \beta) \frac{\ln \left|F\left(z_{\alpha}^{\prime}\right)\right|}{\ln \mu(\sigma)}, \quad 0<\beta \leqslant \frac{1}{5}
$$

Так как $\left|\operatorname{Re} z_{\alpha}^{\prime}-\sigma\right| \leqslant \beta^{-1} h$, учитьвая еще раз оценки (43), отсюда окончательно получаем, что

$$
\varlimsup_{\substack{\sigma \in e_{\beta} \\ \sigma \rightarrow \infty}} \frac{\ln \mu^{*}(\sigma)}{\ln \mu(\sigma)} \leqslant 2 \beta+(1-2 \beta) d(F ; \gamma),
$$

где $0<\beta \leqslant 1 / 5, e_{\beta}=[0, \infty) \backslash E_{\beta}$.

Теорема 1 полностью доказана. 
4. Доказательство теоремы 2. Достаточность. Последовательность $\left\{Q^{\prime}\left(\lambda_{n}\right)\right\}$ $\bar{W}$-нормальна. Следовательно, существует $\theta \in L$ такая, что

$$
\lim _{x \rightarrow \infty} \frac{1}{\ln x} \int_{1}^{x} \frac{\theta(t)}{t^{2}} d t=0, \quad-\ln \left|Q^{\prime}\left(\lambda_{n}\right)\right| \leqslant \theta\left(\lambda_{n}\right), \quad n \geqslant 1 .
$$

Положим $w(x)=\max (N($ et $), \theta(t), \sqrt{t})$. Ясно, что $w \in \underline{W}$. Но тогда существует функция $w_{2} \in \underline{W}$ такая, что $w(x)=o\left(w_{2}(x)\right), x \rightarrow \infty$. Пусть $v=v(\sigma)$ - решение уравнения $w_{2}(v)=3 \ln \mu(\sigma)$, где $\mu(\sigma)$ - максимальньй член ряда (2). Из теоремы 1 следует, что для любых $\beta, 0<\beta \leqslant 1 / 5$, сушествует множество $E_{\beta} \subset[0, \infty), d E_{\beta}=0$, такое, что

$$
\varlimsup_{\substack{\sigma \in e_{\beta} \\ \sigma \rightarrow \infty}} \frac{\ln \mu^{*}(\sigma)}{\ln \mu(\sigma)} \leqslant 2 \beta+(1-2 \beta) d(F ; \gamma),
$$

где $e_{\beta}=[0, \infty) \backslash E_{\beta}$.

Пусть $k=k(\sigma)$ - центральный индекс измененного ряда

$$
F^{*}(s)=\sum_{n=1}^{\infty} a_{n} Q^{\prime}\left(\lambda_{n}\right) e^{\lambda_{n} s}
$$

В ходе доказательства теоремы 1 было установлено, что $\lambda_{k(\sigma)} \leqslant v(\sigma)$ для $\sigma \in e_{\beta}$. Следовательно, для $\sigma \in e_{\beta}$

$$
\mu(\sigma) \leqslant \mu^{*}(\sigma)\left|\frac{1}{Q^{\prime}\left(\lambda_{n}\right)}\right| \leqslant \mu^{*}(\sigma) e^{\theta(v(\sigma))}=\mu^{*}(\sigma) \mu(\sigma)^{o(1)} .
$$

Так что для $\sigma \in e_{\beta}$ и $\sigma \rightarrow \infty$

$$
(1+o(1)) \ln \mu(\sigma) \leqslant \ln \mu^{*}(\sigma) .
$$

Но тогда из (44) следует, что

$$
1 \leqslant 2 \beta+(1-2 \beta) d(F ; \gamma) \leqslant 1, \quad 0<\beta \leqslant \frac{1}{5}
$$

Устремляя $\beta$ к нулю, отсюда окончательно получим равенство $d(F ; \gamma)=1$. Следовательно, $d(F)=1$.

Необходимость. Будем доказывать от противного. Пусть для любой функции $F \in$ $D(\Lambda, R)$ выполняется равенство $d(F)=1$, но

$$
\underset{x \rightarrow \infty}{\lim _{x \rightarrow \infty}} \frac{1}{\ln x} \sum_{\lambda_{n} \leqslant x} \frac{1}{\lambda_{n}}>0 .
$$

Рассмотрим функцию

$$
F(s)=\sum_{n=1}^{\infty} \frac{1}{\left(1+\lambda_{n}\right)^{2}} \frac{\varphi^{2}\left(\lambda_{n}\right)}{Q^{\prime}\left(\lambda_{n}\right)} e^{\lambda_{n} s}, \quad s=\sigma+i t
$$


где

$$
\varphi(\lambda)=\prod_{n=1}^{\infty}\left(1+\frac{\lambda}{\lambda_{n}}\right) e^{-\lambda / \lambda_{n}}, \quad Q(\lambda)=\prod_{n=1}^{\infty}\left(1-\frac{\lambda^{2}}{\lambda_{n}^{2}}\right) .
$$

Поскольку $n(x)=O(x), x \rightarrow \infty$, то из (45) следует, что [10]

$$
\ln \varphi(x) \leqslant-d x \ln x+d, \quad x>0,
$$

где $0<d<\infty$. Следовательно, ряд (46) абсолютно сходится во всей плоскости и $R$-порядок его суммы не превосходит $d^{-1}<\infty$. В работе [10] показано, что $|F(\sigma)| \leqslant$ $M<\infty$ для $0 \leqslant \sigma<\infty$. Это означает, что $d(F ;[0, \infty)) \leqslant 0$. Так что $d(F) \leqslant 0$. Получили противоречие. Следовательно,

$$
\varliminf_{x \rightarrow \infty} \frac{1}{\ln x} \sum_{\lambda_{n} \leqslant x} \frac{1}{\lambda_{n}}=0 .
$$

Теорема 2 полностью доказана.

\section{СПИСОК ЦИТИРОВАННОЙ ЛИТЕРАТУРЫ}

[1] Леонтьев А. Ф. Ряды экспонент. М.: Наука, 1976.

[2] Леонтьев А. Ф. Последовательности полиномов из экспонент. М.: Наука, 1980.

[3] Гайсин А. М. Оценка роста и убывания целой функции бесконечного порядка на кривых // Матем. сб. 2003. Т. 194. № 8. С. 55-82.

[4] Beurling A. Some theorems on boundedness of analytic functions // Duke Math. J. 1949. V. 16. P. $355-359$.

[5] Гольдберг А. А., Островский И. В. Распределение значений мероморфных функций. М.: Наука, 1970.

[6] Гайсин А. М., Латыпов И. Д. Асимптотика логарифма максимального члена измененного ряда Дирихле // Изв. вузов. Матем. 2002. № 9(484). С. 15-24.

[7] Pólya. G. Untersuchungen über Lücken und Singularitäten von Potenzreihen // Math. Z. 1929. V. 29. P. 549-640.

[8] Шеремета М.Н. О росте на действительной оси целой функции, представленной рядом Дирихле // Матем. заметки. 1983. Т. 33. № 2. С. 235-245.

[9] Гайсин А. М. Поведение суммы ряда Дирихле заданного роста // Матем. заметки. 1991. Т. 50. № 8 . С. $47-56$.

[10] Гайсин А. М. Об одной гипотезе Полиа // Изв. РАН. Сер. матем. 1994. Т. 58. № 2. С. $73-92$.

[11] Хейман У. Мероморфные функции. М.: Мир, 1966.

(А. М. Гайсин) Институт математики с ВЦ Уфимского научного центра РАН

Поступило

(И. Д. Латыпов) Башкирский государственный университет, г. Уфа

16.08.2004

E-mail: gaisin@imat.rb.ru, latypovid@yandex.ru 